\title{
PSEUDOSPECTRUM AND CONDITION SPECTRUM
}

\author{
G. KRISHNA KUMAR AND S. H. LUI
}

Abstract. For $0<\varepsilon<1$, the $\varepsilon$-condition spectrum of an element $A \in \mathbb{C}^{N \times N}$, a generalization of eigenvalues, is denoted by $\sigma_{\varepsilon}(A)$, and is defined as ([7]),

$$
\sigma_{\varepsilon}(A):=\left\{z \in \mathbb{C}: z I-A \text { is not invertible or }\|z I-A\|\left\|(z I-A)^{-1}\right\| \geqslant \frac{1}{\varepsilon}\right\} .
$$

Several results on spectrum and $\varepsilon$-pseudospectrum are generalized to $\varepsilon$-condition spectrum. The $\varepsilon$-condition spectrum is a useful tool in the numerical solution of operator equations. In [3], the authors have given an analogue of the Spectral Mapping Theorem for condition spectrum. This paper is a continuation of the papers [5] and [3], generalizing the Spectral Mapping Theorem for eigenvalues. In this paper we are studying size of the components of condition spectrum of a matrix. The main contribution of this paper consists of asymptotic expansions of quantities which determine the size of components of condition spectral sets. A relation connecting pseudospectrum and condition spectrum of a matrix is given as set inclusions. Using this relation a weak version of component wise condition Spectral Mapping Theorem is given. Examples are given to illustrate the theory developed.

Mathematics subject classification (2010): 47A10, 47A11, 65F15, 65F35.

Keywords and phrases: Eigenvalues, pseudospectrum, condition spectrum, spectral mapping theorem.

\section{REFERENCES}

[1] M. KAROw, Eigenvalue condition numbers and a formula of Burke, Lewis and Overton, Electron. J. Linear Algebra 15 (2006), 143-153.

[2] G. KRISHNA KUMAR AND S. H. KULKARNI, Linear maps preserving pseudospectrum and condition spectrum, Banach J. Math. Anal. 6, 1 (2012), 45-60.

[3] G. Krishna Kumar and S. H. Kulkarni, An Analogue of the Spectral Mapping Theorem for Condition Spectrum, Operator Theory: Advances and Applications, Vol. 236, 299-316.

[4] S. H. LuI, A pseudospectral mapping theorem, Math. Comp. 72, 244 (2003), 1841-1854 (electronic).

[5] S. H. LuI, Pseudospectral mapping theorem II, Electron. Trans. Numer. Anal. 38 (2011), 168-183.

[6] W. Rudin, Functional analysis, McGraw-Hill, New York, 1973.

[7] S. H. Kulkarni And D. Sukumar, The condition spectrum, Acta Sci. Math. (Szeged) 74, 3-4 (2008), 625-641.

[8] T. J. RANSFORD, Generalized spectra and analytic multivalued functions, J. London Math. Soc. (2) 29, 2 (1984), 306-322.

[9] L. N. Trefethen and M. Embree, Spectra and pseudospectra, Princeton Univ. Press, Princeton, NJ, 2005. 